\title{
INFLUENCE OF HbA1c IN TOTAL KNEE ARTHROPLASTY WOUND HEALING
}

\author{
B. Narendra Reddy1, P. Anil Babu²
}

${ }^{1}$ Associate Professor, Department of Orthopaedics, NRI Medical College, Chinakakani, Guntur, Andhra Pradesh. ${ }^{2}$ Assistant Professor, Department of Orthopaedics, GMC, Guntur, Andhra Pradesh.

\section{ABSTRACT}

\section{BACKGROUND}

Wound healing can be at risk in Diabetic patients undergoing any surgical procedure. The operative procedures done in elderly patients are at higher risk for developing post-operative complications including wound healing. Though the patients claim and get evaluated for Diabetes mellitus with normal or under control Blood sugar levels, HbA1c levels are very often elevated indicating the fact that the normal or under control Blood sugar levels are with previous long-term poor controls.

\section{MATERIALS AND METHODS}

In a study group of 280 patients with poor glycaemic control, undergoing a major surgery, the influence of elevated HbA1c is studied for the wound healing based on Hollander Wound Evaluation Score (HWES).

\section{RESULTS}

All the patients in the study group have good HWES on or before first month of followup.

\section{CONCLUSION}

Though poor glycaemic control is known by elevated HbA1c, it alone cannot influence the wound healing in the presence of corrected and maintained blood sugar levels.

\section{KEYWORDS}

Total Knee Arthroplasty, Complications, Wound Healing, HbA1c.

HOW TO CITE THIS ARTICLE: Reddy BN, Babu PA. Influence of HbA1c in total knee arthroplasty wound healing. J. Evolution Med. Dent. Sci. 2017;6(46):3561-3564, DOI: 10.14260/Jemds/2017/767

\section{BACKGROUND}

Wound healing can be at risk in Diabetic patients undergoing any surgical procedure. The operative procedures done in elderly patients are at higher risk for developing postoperative complications including wound healing. Certain surgeries done predominantly in elderly age group are to be planned in such a way that proper wound healing should be ensured, right from the beginning at pre-operative evaluation. One such risk factor is diabetes mellitus with preexisting Type 2 diabetes mellitus, which is increasingly found in elderly patients opting to undergo Total Knee Arthroplasty (TKA). Though the patients claim and get evaluated for Diabetes mellitus with normal or under control Blood sugar levels, HbA1c levels are very often elevated indicating the fact that the normal or under control Blood sugar levels are with previous long-term poor controls.(1)

As there are many other factors that can influence wound healing like smoking, vascular deficits, some of them are not found in most of the patients. In patients undergoing same surgery with similar technique, the effect of long-term poor control of diabetes mellitus needs to be studied with reference to elevated HbA1c levels. Proper wound healing is very essential in TKA like any other surgery to avoid complications and for early mobilisation.(2)

Financial or Other, Competing Interest: None.

Submission 30-04-2017, Peer Review 25-05-2017,

Acceptance 01-06-2017, Published 08-06-2017.

Corresponding Author:

Dr. P. Anil Babu,

D. No. 5-54-120, 101 Suryodaya Residency,

6/18 Brodipet, Guntur-522002,

Andhra Pradesh, India.

E-mail: anilpbabu@gmail.com

DOI: $10.14260 /$ jemds $/ 2017 / 767$
The poor glycaemic control as indicated by elevated HbA1c needs to be studied that whether it can be an influencing factor in the presence of controlled blood sugar level during the post-operative period following Total Knee Arthroplasty (TKA). As it has been studied in different surgeries that the elevated HbA1c may not influence the wound healing, a major surgery like Total Knee Arthroplasty needs to be studied. Following TKA, implant and bone interface can have aseptic loosening, leading to stress lines at the interfaces. Wound healing following TKA is very much essential for the survival of the implant in the given bone cut, failure of which will hamper the better functional outcomes. ${ }^{(3,4)}$

Lower limb surgeries, especially are prone for developing post-operative complications with wound dehiscence and subsequent infections.(5) Elevated $\mathrm{HbA1c}$ in such cases essentially indicate that poor glycaemic controls are more than three months old. Every case with elevated levels must be considered as a case for maintaining blood glucose levels in the post-operative period with utmost care, to avoid lot of post-operative complications related to wound healing.

Hollander Wound Evaluation Score (HWES) is a validated and widely used cosmetic scoring system for post-operative wound healing. This score consisted of a cosmetic score from 0 (worst result) to 6 (best result) based on the presence or absence of six features, namely step-off of borders, contour irregularities, margin separation, edge inversion, excessive distortion and overall appearance.

When the factors like built, blood haemoglobin, normal renal, hepatic profiles, thyroid status, primary osteoarthritis as an indication for TKA done by a single surgeon, in single centre with single approach are considered with one adverse 
factor like poor glycaemic control, can be studied for causing any wound healing problems.

The wound healing following TKA can be evaluated by applying HWES to know the influence of HbA1c at the onemonth followup of post-operative cases.

\section{MATERIALS AND METHODS}

We have done this study to know the influence of poor glycaemic controls on patients undergoing Total Knee Arthroplasty. The study is designed as an observational study to know the influence of HbA1c in Total Knee Arthroplasty wound healing, when all other factors that can influence wound healing are not present. Our study group consists of 280 patients of primary osteoarthritis of knee joint planned for Arthroplasty. The inclusion criterion are cases of primary osteoarthritis, > $10 \mathrm{gm}$ of Haemoglobin levels, normal Thyroid, Renal, Liver profiles, no clinical signs of neurovascular deficits, Blood sugar levels under control with either hypoglycaemic agents or Mixtard insulin; and poor glycaemic controls with $\mathrm{HbA1c}>7 \%$.

The exclusion criteria are secondary osteoarthritis for any reason, previous surgical or trauma wounds on the anterior aspect of the knee, uncontrolled diabetes mellitus or preexisting vascular deficits.

The cases in the selected group has undergone TKA by a single surgeon with single approach with anterior midline skin incision, medial parapatellar approach with implants with posterior stabilised design. These cases are given one dose of ceftriaxone and Tranexamic acid preoperatively. The wounds are closed with suction drain with polyglactin 910 for patellar tendon and subcutaneous tissue and staples for skin. Mild Robertson Jones compression bandage is applied for two days.

Post-operatively, six doses of Cefuroxime, four doses of tranexamic acid are given with pain management by NSAIDs. The drain is removed on second post-operative day. The patients are discharged on fourth post-operative day without any antibiotics. Patients are given two dressing changes for the surgical wound, before the sutures are removed from $11^{\text {th }}$ to $14^{\text {th }}$ post-operative day.

Post-operatively, the quality of the wound is assessed by applying Hollander Wound Evaluation Score (HWES) at the suture removal and on first month followup. The wounds are given scores ranging from 0 to 6 , where 0 being worst and 6 for the best; based on the presence or absence of six features, namely step-off of borders, contour irregularities, margin separation, edge inversion, excessive distortion and overall appearance.

The statistical tests are done in this cohort of all the cases with elevated HbA1c levels pre-operatively and statistical analysis is done by calculating chi-square test and the significance with $\mathrm{P}$ value for the findings of the study group with HWES for patients having HbA1c levels, indicating moderately and very high values, indicating the severity or poor glycaemic control.

\section{RESULTS}

The study group has a scar measuring 8.5 to $12 \mathrm{cms}$. The results are assessed by applying Hollander Wound Evaluation Score (HWES) during the post-operative reviews on suture removal and on first month followup. The scoring system consists of a cosmetic score from 0 (Worst result) to 6 (Best result) based on the presence (0) or absence (1) of six features, namely step-off of borders, contour irregularities, margin separation, edge inversion, excessive distortion and overall appearance. In our study HWES showed one feature, excessive distortion in 17 cases, which improved to score 1. Rest of the features considered in the scoring system remained at 1 during the two evaluations done in 261 cases.

The reason for the excessive distortion to be improved is the range of movements that are regained by the patient, resulting in the improvement to normal scar without distortion.

\section{Analysis}

The study group consists of 280 primary osteoarthritis patients comprising of 112 male and 168 female patients. The mean Haemoglobin level of the cases is $10.2 \mathrm{gm} \%$. The mean blood sugar level is Fasting Blood sugar at $104 \mathrm{mg} \%$, and Random Blood sugar at $132 \mathrm{mg} \%$ with history of diabetes mellitus with poor controls for periods spanning 2 to 5 years prior to pre-operative evaluation for TKA. The number of patients on oral hypoglycaemic agents are 158 and on Mixtard insulin are 122.

The HbA1c levels are taken pre-operatively. The study group has these levels ranging from $7 \%$ to $9 \%$, indicating poor glycaemic controls. The study group further divided into two groups one with $\mathrm{HbA1c}$ levels between $7 \%$ to $8 \%$ and other group with levels $>8.1 \%$.

Each group with elevated HbA1c levels showed the following group specific HWES. In the group with HbA1c within $7 \%$ to $8 \%$, scores of 6 at suture removal are seen in 155 cases and at one month in 6 cases. In the group with HbA1c with $>8.1 \%$, scores of 6 at suture removal are seen in 108 cases and at one month in 11 cases.

The Chi-squared statistic and $p$ values are calculated. The observed values, (expected cell values), [Chi-square statistic for each value], are given in columns. The Chi-squared statistic for the study chi-squared statistic is 3.652 . The pvalue is .056002 . This result is not significant at $\mathrm{p}<.05$.

\begin{tabular}{|c|c|c|c|}
\hline & $\begin{array}{c}\text { HWES 6 at } \\
\text { SR }\end{array}$ & $\begin{array}{c}\text { HWES 6 at } \\
\text { One Month }\end{array}$ & $\begin{array}{c}\text { Marginal } \\
\text { Row Totals }\end{array}$ \\
\hline $\begin{array}{c}\text { HbA1c 7\% } \\
\text { to 8\% }\end{array}$ & $\begin{array}{c}155(151.22) \\
{[0.09]}\end{array}$ & $\begin{array}{c}6(9.78) \\
{[1.46]}\end{array}$ & 161 \\
\hline $\begin{array}{c}\mathrm{HbA} 1 \mathrm{c}> \\
8.1 \%\end{array}$ & $\begin{array}{c}108(111.78) \\
{[0.13]}\end{array}$ & $\begin{array}{c}11(7.22) \\
{[1.97]}\end{array}$ & 119 \\
\hline $\begin{array}{c}\text { Marginal } \\
\text { Column } \\
\text { Totals }\end{array}$ & $\mathbf{1 7}$ & $\begin{array}{c}\mathbf{2 8 0} \\
\text { (Grand } \\
\text { Total) }\end{array}$ \\
\hline \multicolumn{3}{|c|}{ Table 1. Statistical Analysis of Wound Healing with } \\
Elevated HbA1c Levels in TKA \\
\hline
\end{tabular}

The Chi-squared statistic is 3.652 . The p value is .056002 . This result is not significant at $\mathrm{p}<.05$. 


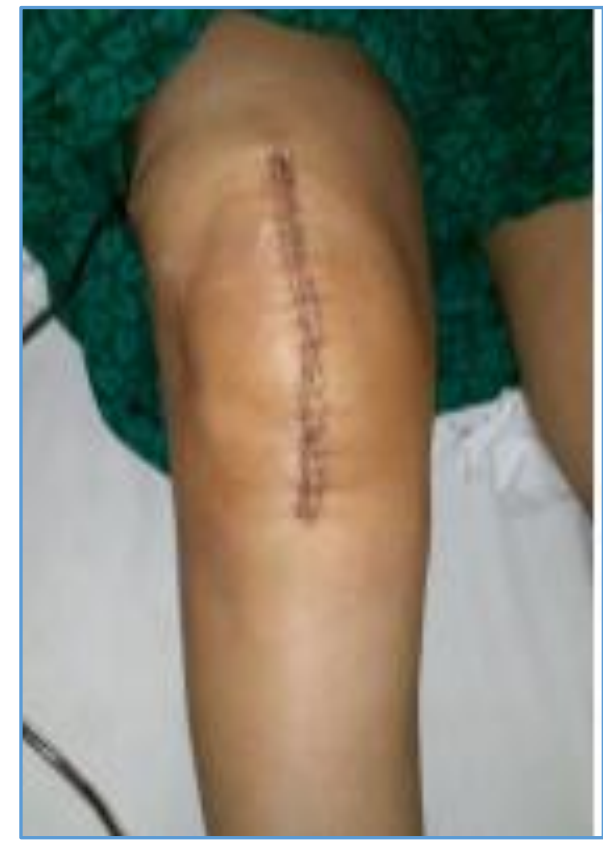

Figure 1. Second POD

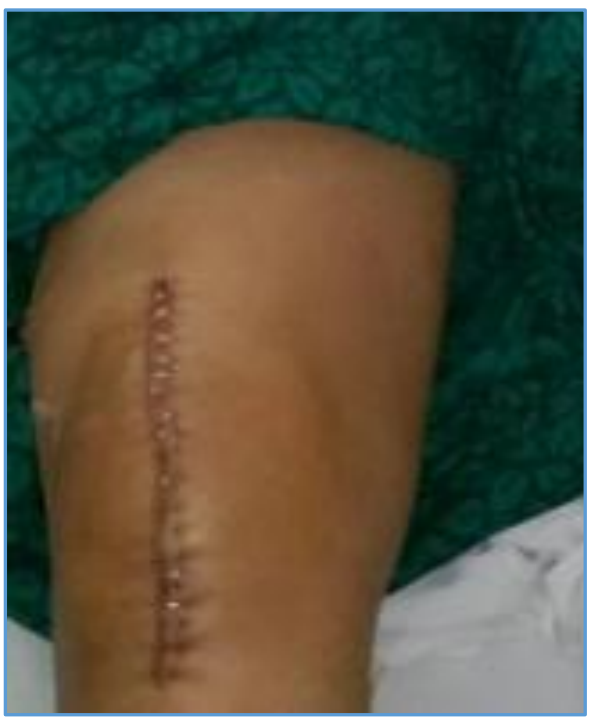

Figure 2. Fourth POD

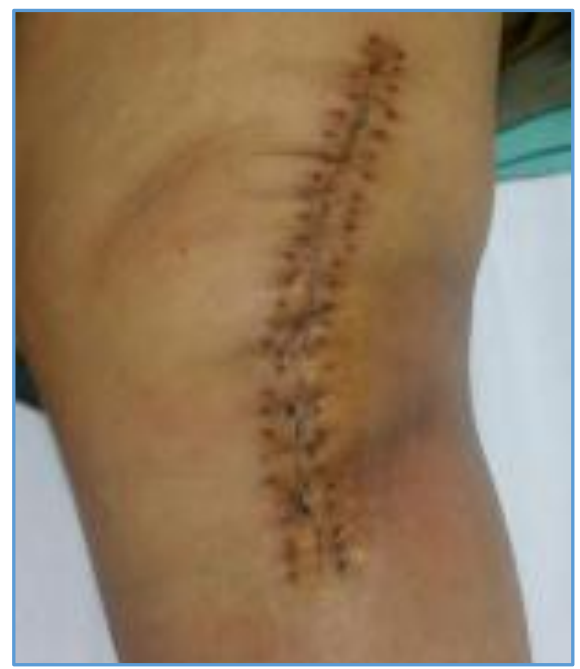

Figure 3. Suture Removal- Distortion

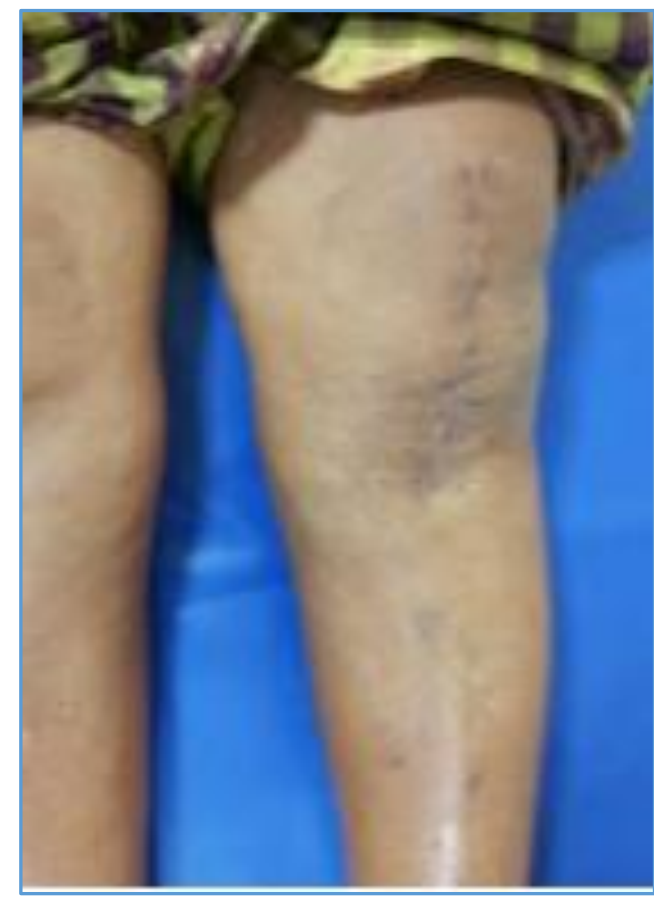

Figure 4. One-Month Followup

\section{DISCUSSION}

Lower limb wounds can be complicated by diabetes. Haemoglobin A1c (HbA1c) reflects glycaemia over 2 - 3 months and is the standard measure used to monitor glycaemia in diabetic patients, but results from studies have not shown a consistent association of HbA1c with wound healing.(6) When perfect wound healing is expected in TKA, all the risk factors which can hamper the wound healing need to be evaluated. Patients who are evaluated pre-operatively for an elective surgery like TKA, when found with elevated HbA1c levels are at risk with uncontrolled diabetes. Wound healing is an essential part of TKA, which can determine the outcome. Poor wound healing, wound dehiscence not only delay the physiotherapy for regaining range of movements, but also lead to perioperative complications. There are many factors that can influence the wound healing in a major surgery like Total Knee Arthroplasty. In the presence of a major risk factor like Diabetes mellitus which can influence the wound healing, the elevated level of HbA1c indicate poor glycaemic control in the immediate past history.

All the risk factors for poor wound healing can occur simultaneously. The presence of elevated HbA1c levels indicate that absolute diabetic control is required peroperatively to avoid many complications. The HbA1c in elevated levels may indicate poor glycaemic control previously, but with proper maintenance of normal blood sugar levels, wound healing is achieved in TKA.

\section{CONCLUSION}

When good control of Blood sugar levels are achieved in immediate pre-operative and post-operative periods, the presence of $\mathrm{HbA1c}$ alone cannot influence the wound healing. It is the holistic approach that can assure better wound healing rather than an unelevated HbA1c levels alone. Lot of issues need to be carefully planned, viz. surgery under antibiotic cover, pre- and post-operative monitoring of risk factors. 


\section{REFERENCES}

[1] Okamura A, Watanabe M, Imamura Y, et al. Preoperative glycosylated hemoglobin levels predict anastomotic leak after esophagectomy with cervical esophagogastric anastomosis. World J Surg 2016;41(1):200-7.

[2] Harato K, Tanikawa H, Morishige $Y$, et al. What are the important surgical factors affecting the wound healing after primary total knee arthroplasty? Journal of Orthopaedic Surgery and Research 2016;11(1):7.

[3] Fernandes KS, Glick M, de Souza MS, et al. Association between immunologic parameters, glycemic control, and post extraction complications in patients with type 2 diabetes. Am Dent Assoc 2015;146(8):592-9.
[4] Oates TW, Galloway P, Alexander P, et al. The effects of elevated hemoglobin $\mathrm{A}(1 \mathrm{c})$ in patients with type 2 diabetes mellitus on dental implants: survival and stability at one year. J Am Dent Assoc 2014;145(12):1218-26.

[5] Humphers JM, Shibuya N, Fluhman BL, et al. The impact of glycosylated hemoglobin and diabetes mellitus on wound-healing complications and infection after foot and ankle surgery. Am Podiatr Med Assoc 2014;104(4):320-9.

[6] Christman AL, Selvin E, Margolis DJ, et al. Hemoglobin A1c predicts healing rate in diabetic wounds. J Invest Dermatol 2011;131(10):2121-7. 